\title{
Risk Factors for and the Prevalence of Peripheral Arterial Disease and its Relationship to Carotid Atherosclerosis: The Kyushu and Okinawa Population Study (KOPS)
}

\author{
Hachiro Ohnishi ${ }^{1}$, Yasunori Sawayama², Norihiro Furusyo ${ }^{1,2}$, Shinji Maeda ${ }^{2}$, Shoji Tokunaga ${ }^{3}$, \\ and Jun Hayashi ${ }^{1,2}$
}

${ }^{1}$ Department of Environmental Medicine and Infectious Diseases, Faculty of Medical Sciences, Kyushu University, Fukuoka, Japan ${ }^{2}$ Department of General Internal Medicine, Kyushu University Hospital, Fukuoka, Japan

${ }^{3}$ Department of Medical Informatics, Kyushu University Hospital, Fukuoka, Japan

\begin{abstract}
Aim: Peripheral arterial disease (PAD) is associated with cerebrovascular disease, ischemic heart disease, and other cardiovascular disease. We investigated the prevalence of and factors related to PAD to clarify the relationship between PAD and carotid atherosclerosis in a cross-sectional populationbased study.

Methods: The study included 2,402 (900 males and 1,502 females; mean $\pm \mathrm{SD}=64.9 \pm 10.9$ years) of 3,862 residents of two Japanese rural areas who reported for a free health examination in 2005 or 2006. An ankle brachial index value $\leq 0.9$ was considered to be PAD. The carotid artery intima-media thickness (CA-IMT) was measured by carotid ultrasound.

Results: The prevalence of PAD was $1.71 \%(n=41)$ of all participants. The risk factors independently associated with a significantly higher risk of PAD, identified by multivariate analysis, are as follows: For males, age, dyslipidemia, and CA-IMT, and for females, age, waist circumference, and dyslipidemia.

Conclusion: The prevalence of PAD in Japan was confirmed to be lower than that of similar studies performed in other countries. PAD was strongly correlated with age and dyslipidemia in both sexes, carotid atherosclerosis in males, and abdominal fat in females.
\end{abstract}

J Atheroscler Thromb, 2010; 17:751-758.

Key words; Carotid atherosclerosis, Epidemiology, Intima-media thickness, Peripheral arterial disease, Waist circumference

\section{Introduction}

Japanese lifestyle changes have led to the recent increase of atherosclerosis, which is related to hypertension, dyslipidemia, glucose intolerance, and obesity, which are also increasing ${ }^{1-3)}$. Many risk factors are involved in the occurrence of atherosclerosis, which manifests as cerebrovascular disease, coronary artery disease, and peripheral arterial disease $(\mathrm{PAD})^{4,5)}$.

Address for correspondence: Jun Hayashi, M.D., Ph.D., Professor, Department of General Internal Medicine, Kyushu University Hospital, Fukuoka, 812-8582, Japan

E-mail: hayashij@gim.med.kyushu-u.ac.jp

Received: August 27, 2009

Accepted for publication: December 18, 2009
The signs and symptoms of PAD are usually thought to progress slowly in the natural progression of atherosclerosis. Atherosclerosis disease of the peripheral arteries is considered to be a particularly serious problem for patients because of reduced physical activity and quality of life, and shortened lifespan. $\mathrm{PAD}$ is associated with the occurrence of coronary artery disease, and stroke ${ }^{5-7)}$. Thus, PAD should be detected and treated as early as possible.

Many large-scale clinical research trials for arterial sclerosis have been performed, and reports of epidemiological surveys about PAD can be found from the United States, Europe and Asia ${ }^{5,8-11)}$, however, there have been few published epidemiological studies of PAD in Japan ${ }^{12-14)}$. 
Measurement of carotid artery intima-media thickness (CA-IMT) by high-resolution B-mode carotid ultrasound examination has been used as a noninvasive method for detecting early carotid atherosclerosis. We have examined and reported on CA-IMT in a number of areas of southwestern Japan ${ }^{2,3)}$. In previous studies, CA-IMT has been associated with cardiovascular disease and stroke and has shown significant associations with PAD ${ }^{5,6,15-17)}$; however, little is known about the relationship between PAD and CAIMT of Japanese ${ }^{14)}$.

The aim of this study was to investigate the prevalence of and factors related to PAD and to clarify the risk factors for PAD using data gathered at routine health examinations and CA-IMT in southwestern Japan.

\section{Materials and Methods}

\section{Participants}

The basic demographics of the two rural studied areas can be summarized as follows: Iki City, with about 31,400 residents, is an isolated island in southwestern Japan where fishing and farming are the main economic activities ${ }^{18)}$. Hoshino Village, with about 3,400 residents, is a rural, mountainous, agriculture village in Fukuoka prefecture that is relatively isolated from other communities ${ }^{19)}$. The lifestyle in these areas does not differ much from that of other parts of Japan. The participants were residents notified by newspaper and public announcements of a free health examination given by the Department of General Internal Medicine, Kyushu University.

The study included 2,402 (900 male and 1,502 female, age range 40-93, mean $\pm S D=64.9 \pm 10.9$ years) of 3,862 Iki City and Hoshino Village residents who reported for a free health examination in 2005 or 2006. In 2005, the data of 1,417 residents of Iki City were available for analysis, (506 male and 911 female, age $40-93$ years, mean $\pm S D=64.5 \pm 12.5$ years), and in 2006, the data of 985 residents of Hoshino Village were available for analysis, (394 male and 591 female subjects, age $40-92$ years, mean $\pm S D=65.3 \pm 11.7$ years). Excluded were 1,281 residents who did not complete all of the required examinations or who did not return the questionnaire, 156 who were under 40 years of age, 13 with TG levels $>400 \mathrm{mg} / \mathrm{dL}, 8$ with missing ABI measurements, and 2 with $\mathrm{ABI}>1.50$. Exclusion was because of possible measurement artifacts reflecting the presence of rigid or calcified arterial walls $s^{5,7)}$.

To ensure the validity of the data, all doctors participating in the study were members of the General
Internal Medicine Department of Kyushu University who were briefed on the study protocol and trained in the medical techniques necessary for the study. The design of this study was approved by the Kyushu University Hospital ethics committee. Informed consent was obtained from all participants before the examination.

\section{Medical History}

At the baseline examination, anthropometry and blood pressure were measured by public health nurses. Body mass index (BMI) and waist circumference (WC) were adopted as indicators of obesity. Height and weight were measured with light clothes and without shoes. BMI was calculated as weight (in kilograms) divided by height (in meters) squared. WC was measured in the horizontal plane at the umbilical level. Smoking behavior was assessed by questionnaire, and the subjects were classified as smokers (current or past smokers) or non-smokers. Systolic blood pressure (SBP) and diastolic blood pressure (DBP) were measured at rest in a sitting position. SBP above 140 $\mathrm{mmHg}$ and/or DBP above $90 \mathrm{mmHg}$ or a subject taking anti-hypertensive agents were defined as hypertension (HT). Fasting plasma glucose (FPG) above 110 $\mathrm{mg} / \mathrm{dL}$ and/or treatment for diabetes was defined as glucose intolerance. Total cholesterol (TC) above 220 $\mathrm{mg} / \mathrm{dL}$, triglycerides (TG) above $150 \mathrm{mg} / \mathrm{dL}$, and/or high-density lipoprotein cholesterol (HDL-C) below $40 \mathrm{mg} / \mathrm{dL}$, and/or low-density lipoprotein cholesterol (LDL-C) above $140 \mathrm{mg} / \mathrm{dL}$ or lipid-lowering drug administration were defined as dyslipidemia. Chronic kidney disease (CKD) was defined as an estimated creatinine clearance $(\mathrm{eCcr})<60 \mathrm{~mL} / \mathrm{min} / 1.73 \mathrm{~m}^{2}$ (CKD stages $3-5)^{20}$. Estimation of the glomerular filtration rate (eGFR) by the Cockcroft-Gault method was adjusted by the body surface area (BSA) and was used to evaluate kidney function ${ }^{20)}$. The equations are as follows:

Cockcroft-Gault (CG) equation: CG-eGFR $\left(\mathrm{mL} / \mathrm{min} / 1.73 \mathrm{~m}^{2}\right)=\mathrm{Ccr} \times(1.73 / \mathrm{BSA})$ $\mathrm{Ccr}(\mathrm{mL} / \mathrm{min})=((140$-age $) \times$ weight $) /(72 \times$ serum creatinine $) \times(0.85$, if female $)$

BSA $\left(\mathrm{m}^{2}\right)=0.007184 \times$ height $(\mathrm{cm})^{0.725} \times$ weight $(\mathrm{kg})^{0.425}$

\section{Assay Methods}

Blood samples were collected from all participants after overnight fast and stored at $-20^{\circ} \mathrm{C}$ until analysis. The following parameters were measured: FPG, high sensitive C-reactive protein (hs-CRP), serum creatinine and serum levels of TC, TG and HDL-C. The LDL-C level was calculated using the 
Table 1. Prevalence of PAD by sex and age

\begin{tabular}{|c|c|c|c|c|c|c|}
\hline \multirow[b]{2}{*}{ Age group } & \multicolumn{2}{|c|}{ Male } & \multicolumn{2}{|c|}{ Female } & \multicolumn{2}{|c|}{ Male and Female } \\
\hline & N. tested & $\mathrm{PAD}+(\%)$ & N. tested & $\mathrm{PAD}+(\%)$ & N. tested & $\mathrm{PAD}+(\%)$ \\
\hline $40-49$ & 112 & --- & 156 & --- & 268 & --- \\
\hline $50-59$ & 152 & --- & 306 & 1.0 & 458 & 0.7 \\
\hline $60-69$ & 256 & 0.4 & 486 & 1.7 & 742 & 1.2 \\
\hline $70-79$ & 313 & 3.2 & 456 & 1.8 & 769 & 2.3 \\
\hline $80+$ & 67 & 9.0 & 98 & 5.1 & 165 & 6.7 \\
\hline$p$ for trend & & $<0.001$ & & 0.008 & & $<0.001$ \\
\hline Total & 900 & 1.89 & 1,502 & 1.73 & 2,402 & 1.71 \\
\hline
\end{tabular}

Friedewald formula ${ }^{21)}$. Subjects with TG levels $>400$ $\mathrm{mg} / \mathrm{dL}$ were excluded $(n=13)$.

\section{Diagnosis of Peripheral Arterial Disease}

The ankle brachial index (ABI) value is useful for the diagnosis of $\mathrm{PAD}^{5,22}$. $\mathrm{ABI}$ is measured by the ratio of SBP at the ankle and at the arm on each side. Subjects with $\mathrm{ABI} \leq 0.9$ on either side or on both sides were considered to have $\mathrm{PAD}^{22}$. The ratio of SBP in the leg to that in the arm was determined using a new device, the form PWV/ABI: BP-203RPEII (Omron Healthcare Co., Ltd, Japan). This device has been used in other Japanese epidemiological studies $^{12-14)}$.

\section{Ultrasonographic Measurement}

CA-IMT and plaques were measured by carotid ultrasound. High-resolution B-mode ultrasound examination was performed with a $7.5 \mathrm{MHz}$ mechanical sector transducer on the Aloka SSD-2000 (Aloka Co. Ltd., Tokyo, Japan) by four specially trained ultrasound technicians ${ }^{2,3)}$. CA-IMT was defined as the distance between two echogenic lines separated by a hypoechoic or anechoic space, with the outer line corresponding to the medial-adventitial border and the inner line representing the luminal-intimal border. CAIMT was measured at points 20,25 , and $30 \mathrm{~mm}$ proximal to the flow divider on the far wall of the right and left common carotid arteries at the end of the diastolic phase. The CA-IMT was the higher of the values for the right or left carotid artery; thus, we defined the maximum-IMT as CA-IMT. Plaque thickness was incorporated into CA-IMT, and CA-IMT above $1.1 \mathrm{~mm}$ was defined as abnormal ${ }^{2,3)}$.

\section{Statistical Analysis}

The Mann-Whitney U-test and Fisher's exact test were used to analyze the characteristics of the PAD and non-PAD groups. Values are expressed as the mean \pm standard deviation (SD). A two-tailed $p<0.05$ was considered significant.

The odds ratio (OR) and 95\% confidence interval (CI) for the possible risk factors of PAD were estimated by age-adjusted and multivariate-adjusted logistic regression analysis. The multiple logistic regression model was selected by the backward stepwise method. The significance level for addition and removal was set as 0.05 and 0.10 , respectively. Because of the strong association between BMI and WC, these variables were inserted in the separate models as candidate variables; thus, the stepwise model was selected in two ways for each sex.

We further estimated the receiver operating characteristic (ROC) curve and the area under the ROC curve (AUC) using the above models for males and females. The confidence intervals for AUC were estimated by the exact method. All analyses were performed using Stata ver 10.

\section{Results}

The prevalence of PAD in the general population of the two Japanese areas studied is shown by sex and age in Table 1. PAD was found in $41(1.71 \%)$ of 2,402 participants. The prevalence of PAD in males $(1.89 \%)$ and females $(1.73 \%)$ was not significantly different. The prevalence of $\mathrm{PAD}$ increased with advancing age in both sexes.

To clarify the effect of cardiovascular risk factors on PAD and to determine predictive markers for patients with PAD, the conventional risk factors and subject characteristics were compared by sex between participants with and without PAD (Table 2).

In a comparison with non-PAD participants, age $(p<0.0001)$, prevalence of HT $(p=0.03)$ and CKD $(p<0.0001)$, and CA-IMT values $(p<0.0001)$ were significantly higher in male PAD patients. Otherwise, age $(p<0.01)$, WC $(p<0.01)$, prevalence of glucose 
Table 2. Basic characteristics of male and female study participants by PAD status

\begin{tabular}{|c|c|c|c|c|c|c|}
\hline \multirow[b]{2}{*}{ Variables } & \multicolumn{3}{|c|}{ Male } & \multicolumn{3}{|c|}{ Female } \\
\hline & $\operatorname{PAD}(n=17)$ & Non-PAD $(n=883)$ & p-value & $\operatorname{PAD}(n=24)$ & Non-PAD $(n=1,478)$ & $p$-value \\
\hline Age (years) & $77.5 \pm 5.2$ & $64.9 \pm 11.0$ & $<0.0001^{*}$ & $71.0 \pm 9.0$ & $64.6 \pm 10.7$ & $<0.01^{*}$ \\
\hline Study area (Iki, \%) & 76.5 & 55.8 & $0.14^{\dagger}$ & 45.8 & 60.9 & $0.14^{\dagger}$ \\
\hline $\operatorname{BMI}\left(\mathrm{kg} / \mathrm{m}^{2}\right)$ & $22.2 \pm 3.1$ & $23.1 \pm 2.9$ & $0.27^{*}$ & $24.2 \pm 3.9$ & $22.8 \pm 3.2$ & $0.06^{*}$ \\
\hline Waist circumference $(\mathrm{cm})$ & $85.0 \pm 7.3$ & $83.9 \pm 7.8$ & $0.41^{*}$ & $85.0 \pm 12.6$ & $77.8 \pm 9.0$ & $<0.01^{*}$ \\
\hline Current + Past smoking (\%) & 64.7 & 64.3 & $1.00^{\dagger}$ & 4.2 & 4.0 & $1.00^{\dagger}$ \\
\hline Current smoking (\%) & 11.8 & 27.0 & $0.27^{\dagger}$ & 4.2 & 2.4 & $0.45^{\dagger}$ \\
\hline $\mathrm{HT}(\%)$ & 76.5 & 49.2 & $0.03^{\dagger}$ & 50.0 & 49.1 & $1.00^{\dagger}$ \\
\hline Glucose intolerance (\%) & 35.3 & 18.6 & $0.11^{\dagger}$ & 29.2 & 10.4 & $0.01^{\dagger}$ \\
\hline Dyslipidemia (\%) & 64.7 & 41.1 & $0.08^{\dagger}$ & 79.2 & 54.7 & $0.02^{\dagger}$ \\
\hline CKD $(\%)$ & 47.1 & 10.8 & $<0.001^{\dagger}$ & 12.5 & 7.0 & $0.24^{\dagger}$ \\
\hline hs-CRP (mg/dL) & $0.12 \pm 0.16$ & $0.09 \pm 0.12$ & $0.16^{*}$ & $0.12 \pm 0.15$ & $0.07 \pm 0.10$ & $<0.01^{*}$ \\
\hline CA-IMT $(\mathrm{mm})$ & $2.21 \pm 0.84$ & $1.23 \pm 0.71$ & $<0.0001^{*}$ & $1.16 \pm 0.52$ & $1.03 \pm 0.49$ & $0.07^{*}$ \\
\hline
\end{tabular}

Abbreviations: BMI: body mass index, HT: hypertension, CKD: chronic kidney disease, hs-CRP: high sensitive C-reactive protein, CA-IMT: carotid artery intima-media thickness

*Mann-Whitney U analysis 'Fisher's exact test

Table 3. Odds ratios for PAD by age-adjusted and stepwise multivariate-adjusted logistic regression analysis

\begin{tabular}{|c|c|c|c|c|c|c|c|c|c|c|c|}
\hline \multirow[b]{3}{*}{ Variablesa } & \multicolumn{5}{|c|}{ Male } & \multicolumn{6}{|c|}{ Female } \\
\hline & \multicolumn{3}{|c|}{ Age-adjusted } & \multicolumn{2}{|c|}{ Multivariate-adjusted ${ }^{*},{ }^{* *}$} & \multicolumn{2}{|c|}{ Age-adjusted } & \multicolumn{2}{|c|}{ Multivariate-adjusted ${ }^{*}$} & \multicolumn{2}{|c|}{ Multivariate-adjusted $^{* *}$} \\
\hline & OR $(95 \% \mathrm{C}$ & C.I.) & p-value & OR (95\% C.I.) & $p$-value & OR (95\% C.I.) & p-value & OR (95\% C.I.) & p-value & OR (95\% C.I.) & p-value \\
\hline Age (per 10 years) & & & & $6.07(2.61,14.12)$ & $<0.0001$ & & & $1.76(1.10,2.81)$ & 0.02 & $1.71(1.08,2.73)$ & 0.02 \\
\hline Study area (Iki vs. Hoshino) & $2.56(0.81$ & $8.15)$ & 0.11 & & & $0.66(0.29, \quad 1.50)$ & 0.32 & & & & \\
\hline BMI $\left(\mathrm{kg} / \mathrm{m}^{2}\right)$ & $1.00(0.83$ & $1.21)$ & 0.96 & & & $1.15(1.03,1.28)$ & 0.01 & & & - & - \\
\hline Waist circumference $(\mathrm{cm})$ & $1.04(0.97$ & $1.11)$ & 0.27 & & & $1.07(1.03, \quad 1.12)$ & $<0.01$ & - & - & $1.07(1.02,1.11)$ & $<0.01$ \\
\hline Current + Past smoking (yes) & $0.97(0.34$ & 2.73) & 0.95 & & & $1.57(0.20,12.12)$ & 0.66 & & & & \\
\hline HT (yes) & $2.12(0.67$ & $6.72)$ & 0.20 & & & $0.71(0.31,1.64)$ & 0.42 & & & & \\
\hline Glucose intolerance (yes) & $2.28(0.80$ & $6.47)$ & 0.12 & & & $2.72(1.09, \quad 6.81)$ & 0.03 & $2.48(1.00,6.16)$ & 0.05 & & \\
\hline Dyslipidemia (yes) & $5.16(1.72, \quad 1$ & $15.44)$ & $<0.01$ & $5.69(1.81,17.87)$ & $<0.01$ & $2.93(1.08,7.89)$ & 0.03 & $2.57(0.95,6.98)$ & 0.06 & $2.67(0.98,7.23)$ & 0.05 \\
\hline CKD (yes) & $1.75(0.53$ & $5.73)$ & 0.35 & & & $0.84(0.22,3.18)$ & 0.80 & & & & \\
\hline hs-CRP (10 times) & $1.44(0.54$ & 3.83) & 0.46 & & & $2.68(1.19,6.03)$ & 0.02 & $2.42(1.05,5.60)$ & 0.04 & & \\
\hline \multicolumn{12}{|l|}{ CA-IMT (mm) } \\
\hline $0.4-1.1$ (low) & 1 (Referen & & & $1 \quad$ (Referent) & & $1 \quad$ (Referent) & & & & & \\
\hline $1.2-1.6$ (moderate) & $8.63(0.98,7$ & $75.91)$ & 0.05 & $2.43(0.93,73.93)$ & 0.06 & $2.43(1.00,5.92)$ & 0.05 & & & & \\
\hline $1.7+$ (high) & $16.98(2.14,13$ & 34.57) & $<0.01$ & $18.09(2.27,144.40)$ & $<0.01$ & $0.69(0.15,3.26)$ & 0.64 & & & & \\
\hline
\end{tabular}

Abbreviations: BMI: body mass index, HT: hypertension, CKD: chronic kidney disease, hs-CRP: high sensitive C-reactive protein, CA-IMT: carotid artery intima-media thickness

*Stepwise model 1: excluding waist circumference ${ }^{* *}$ Stepwise model 2: excluding BMI

intolerance $(p=0.01)$ and dyslipidemia $(p=0.02)$, and hs-CRP values $(p<0.01)$ were significantly higher for female PAD patients.

To separate the risk factors of PAD from confounding factors, we used age-adjusted and stepwise multivariate-adjusted logistic regression analysis (Table 3). In age-adjusted logistic regression analysis, dyslipidemia (OR 5.16, 95\% CI 1.72-15.44) was positively associated with the PAD of males. BMI (OR 1.15, 95\% CI 1.03-1.28), WC (OR 1.07, 95\% CI 1.03-
1.12), glucose intolerance (OR 2.72, 95\% CI 1.096.81), dyslipidemia (OR 2.93, 95\% CI 1.08-7.89), and hs-CRP (OR 2.68, 95\% CI 1.19-6.03) were positively associated with $\mathrm{PAD}$ in females. Compared with the normal CA-IMT (low) group, abnormal CAIMT (moderate) (OR 8.63, 95\% CI 0.98-75.91) and abnormal CA-IMT (high) (OR 16.98, 95\% CI 2.14134.57) were positively associated with $\mathrm{PAD}$ in males. Abnormal CA-IMT (moderate) (OR 2.43, 95\% CI 1.00 -5.92) was positively associated with $\mathrm{PAD}$ in females. 

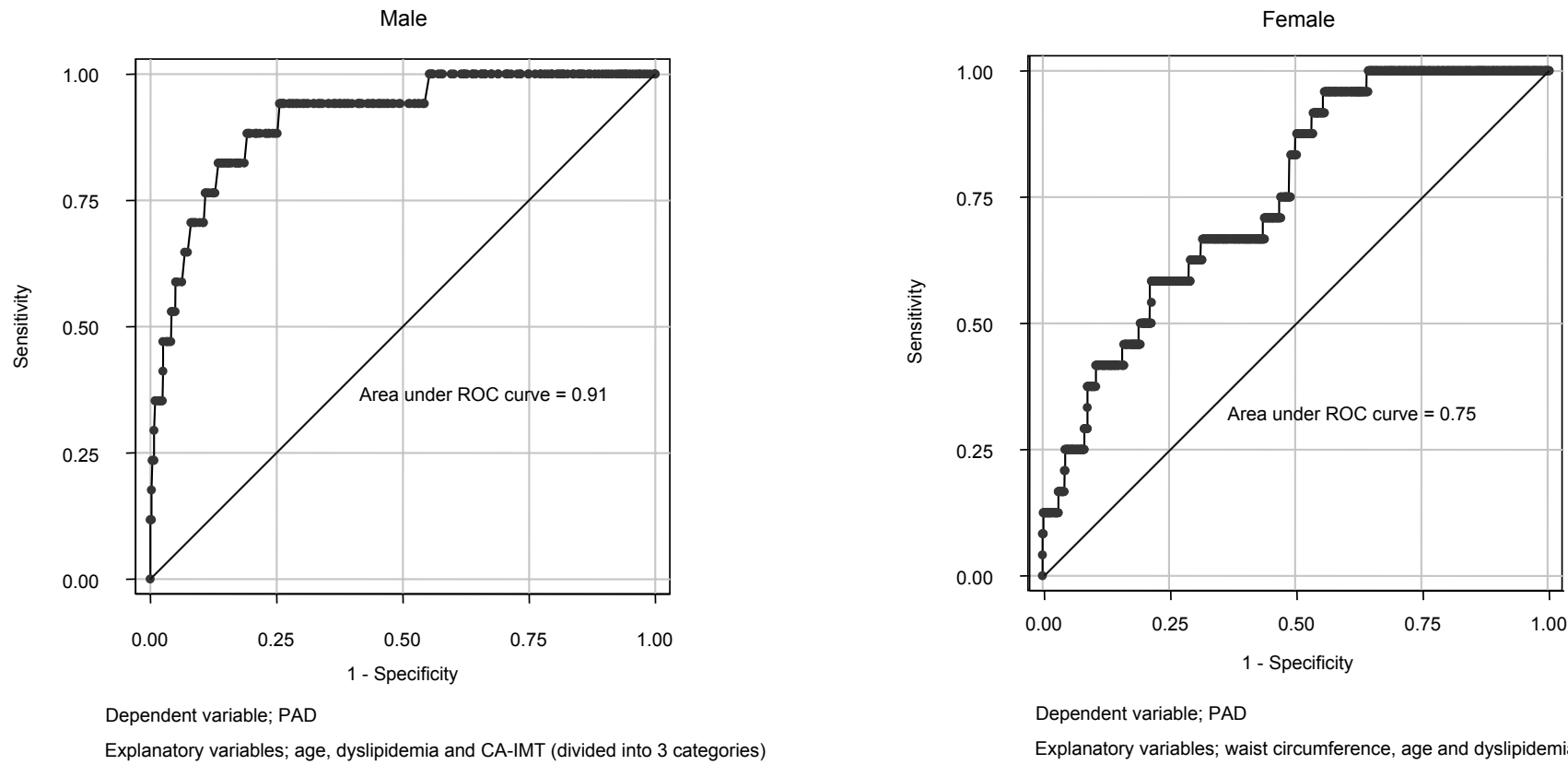

Dependent variable; PAD

Explanatory variables; waist circumference, age and dyslipidemia

Fig. 1.

Receiver operating characteristic curves of PAD from the selected models (Table 3) for 900 male (Fig. 1-a) and 1,052 female (Fig. 1-b) participants. Areas under the receiver operating characteristic curve were 0.91 (95\% CI, 0.89-0.93) for males and 0.75 (95\% CI, 0.73-0.77) for females.

In multivariate-adjusted logistic regression analysis, the stepwise model was selected in two ways for each sex. Because BMI and WC were strongly associated with each other, they were used in separate models as candidate variables. For males, multivariate-adjusted logistic regression analysis results (model 1,2) of age (OR 6.07, 95\% CI 2.61-14.12), dyslipidemia (OR 5.69, 95\% CI 1.81-17.87), and abnormal CA-IMT (high) (OR 18.09, 95\% CI 2.27-144.40) were positively associated with PAD (Table 3). This result was the same when using BMI or WC in the stepwise model. For females, multivariate-adjusted logistic regression analysis results (model 1) of age (OR 1.76, 95\% CI 1.10-2.81), glucose intolerance (OR 2.48, 95\% CI 1.00-6.16), and hs-CRP (OR 2.42, 95\% CI 1.05-5.60), and multivariate-adjusted logistic regression analysis results (model 2) of age (OR 1.71, 95\% CI 1.08-2.73), WC (OR 1.07, 95\% CI 1.02-1.11), and dyslipidemia (OR 2.67, 95\% CI 0.98-7.23) were positively associated with PAD (Table 3).

In order to investigate the validity of the PAD odds ratios, we further calculated the ROC curves and AUC for each sex (Fig. 1). In the application of ROC curves and AUC, data from the stepwise multivariateadjusted logistic regression analysis were used for each sex. As explanatory variables for males, age, dyslipidemia and CA-IMT were used. Age, dyslipidemia, and
WC were used as explanatory variable for females. The AUC (95\% CI) was 0.91 (0.89-0.93) for males (Fig. 1-a) and $0.75(0.73-0.77)$ for females (Fig. 1-b).

\section{Discussion}

In this study, the total prevalence of PAD was $1.71 \%(n=41)$ of all participants. The prevalence of PAD in Japanese studies ${ }^{12-14)}$ has tended to be lower $(1.7-4.3 \%)$ than in studies from other countries (3.6$19.1 \%)^{5,7-11)}$. This is in accordance with the fact that myocardial infarction is found less frequently in Japan than in other countries and indicates that atherosclerosis is less prevalent in Japan. Smoking has been reported to be one of the most important risk factors for PAD and atherosclerosis ${ }^{11)}$, however, our current smoking percentage $(11.5 \%)$ was lower than that of other studies $(15.1-35.8 \%)^{7-11)}$, which may have influenced the low prevalence of PAD found in this study.

Age and dyslipidemia were associated with most of the previously reported conventional risk factors for PAD-related factors ${ }^{5,8-10)}$; however, few studies have reported WC as an independent risk factor for PAD ${ }^{23,24)}$. A previous study reported a relationship between abdominal adiposity and PAD ${ }^{23,24)}$. WC may be associ- 
ated with advanced vascular damage, leading to more generalized atherosclerosis. Several factors may explain the increased prevalence of PAD. First, abdominal adiposity has been associated with significant metabolic abnormalities, including HT, dyslipidemia, glucose intolerance, and insulin resistance ${ }^{25,26}$. Second, abdominal adiposity acts as an endocrine organ by secreting several hormones and cytokines ${ }^{27,28)}$. These adipocytokines are directly or indirectly involved in the process of atherosclerosis. In this study, WC was related to PAD in females, but not in males.

In order to accurately determine the independent factors related to PAD in females, we compared model 1 and model 2. In the first trial, age and dyslipidemia were extracted in both model 1 and model 2, but in the second WC, glucose intolerance, and hs-CRP were selected in both models. A previous study showed that high WC is an obesity indicator related to the association with glucose intolerance ${ }^{25)}$. Some studies have reported that hs-CRP is an independent risk factor for glucose intolerance, and is used as a component of metabolic syndrome ${ }^{29)}$. For all of these reasons, we considered high WC accompanied by hs-CRP and glucose intolerance to be a marker of PAD. Finally, we confirmed age, dyslipidemia, and WC to be independent factors related to PAD in females.

To demonstrate the relationship between PAD and WC, we further analyzed female WC. In the current Japanese metabolic syndrome criteria, WC is defined as $85 \mathrm{~cm}$ for males and $90 \mathrm{~cm}$ for females ${ }^{30)}$; however, this criteria is not based on evidence derived from prospective studies, whereas the Hisayama study suggested that the optimal cutoff point for WC is 90 $\mathrm{cm}$ for males and $80 \mathrm{~cm}$ for females ${ }^{30)}$. For this reason, we compared the cutoff value of the Hisayama criteria with Japanese metabolic syndrome criteria, using model 1 (categorized WC) in females. The odds ratio $(95 \% \mathrm{CI})$ of Hisayama criteria was 2.31 (0.975.51) and its $p$ value was 0.06; Japanese metabolic syndrome criteria were $3.83(1.63-9.00)$ and $p<0.01$ (not shown in Table 3). These results suggest that increased WC has more influence on PAD in females. WC may be a more important marker of PAD and lifestyle-related diseases in females than males.

The Edinburgh Artery Study ${ }^{17)}$ reported a relationship between CA-IMT and PAD for both sexes. Cui et al. ${ }^{14)}$ reported that low ABI $(<0.9)$ was strongly correlated to the internal CA-IMT of elderly male Japanese (sensitivity $65 \%$, specificity $98 \%$ for low ABI to detect CA-IMT $>1.5 \mathrm{~mm}$ ). These results suggested that CA-IMT measurement at health examinations is useful as an indicator of PAD. Our results were mostly consistent with these reports, except for gender differences. We found that age and dyslipidemia are risk factors for PAD and are related to the development of carotid atherosclerosis. Further consideration of carotid atherosclerosis, inflammatory markers and time-dependent change is needed. This study had a cross-sectional design, which means that we can only make assumptions about possible etiologic relationships; therefore, further investigation will be necessary to classify gender differences and the relationship between PAD and CA-IMT.

The area under the ROC curve (AUC) provides a measure of the model's ability to discriminate those who experience the outcome of interest versus those who do not. It is generally accepted, as a general rule, that AUC $\geq 0.9$ is considered as outstanding discrimination, and AUC $\geq 0.7-<0.8$ is considered as acceptable discrimination. The observed AUC of 0.91 in male shows that the male PAD status can be predicted with confidence to some extent by the selected model, and AUC of 0.75 in females suggests that the selected model satisfactorily discriminated the PAD status of females in these residents.

There are some limitations to the present study. First, there may have been some selection bias: the subjects may have been aware of the impact of body weight, smoking, blood pressure, glucose and lipid levels on their health because they had received annual examinations at the health examination center. The prevalence of asymptomatic PAD was high; thus, a larger population-based study will be necessary to clarify the reasons for the high prevalence. Second, in cross-sectional studies it is often difficult to determine the time order of events; therefore, a prospective study should be undertaken to confirm the relationship between PAD and the conventional risk factors for PAD in these residents.

We confirmed that the prevalence of PAD in Japanese was lower than that of similar studies in other countries. The risk factors of PAD in the present study were age and dyslipidemia for both sexes, carotid atherosclerosis for males and abdominal adiposity for females. It is of interest that some of the markers of PAD differed between the sexes.

\section{Acknowledgments}

This study was supported in part by a Grant-inAid for Comprehensive Research of the $21^{\text {st }}$ Century COE Program from the Ministry of Education, Culture, Sports Science and Technology of Japan. 


\section{References}

1) Yamamoto A, Temba H, Horibe H, Mabuchi H, Saito Y, Matsuzawa Y, Kita T, Nakamura H: Life style and cardiovascular risk factors in the Japanese population--from an epidemiological survey on serum lipid levels in Japan 1990 part 2: association of lipid parameters with hypertension. J Atheroscler Thromb, 2003; 10: 176-185

2) Tatsukawa M, Sawayama Y, Maeda N, Okada K, Furusyo N, Kashiwagi S, Hayashi J: Carotid atherosclerosis and cardiovascular risk factors: a comparison of residents of a rural area of Okinawa with residents of a typical suburban area of Fukuoka, Japan. Atherosclerosis, 2004; 172: 337-343

3) Okada K, Maeda N, Tatsukawa M, Shimizu C, Sawayama Y, Hayashi J: The influence of lifestyle modification on carotid artery intima-media thickness in a suburban Japanese population. Atherosclerosis, 2004; 173: 329-337

4) Hoshino A, Nakamura T, Enomoto S, Kawahito H, Kurata H, Nakahara Y, Ijichi T: Prevalence of coronary artery disease in Japanese patients with cerebral infarction: impact of metabolic syndrome and intracranial large artery atherosclerosis. Circ J, 2008; 72: 404-408

5) Zheng ZJ, Sharrett A.R, Chanbless LE, Rosamond WD, Nieto FJ, Sheps DS, Dobs A, Evans GW, Heiss G: Associations of ankle brachial index with clinical coronary heart disease, stroke and preclinical atherosclerosis risk in communities (ARIC) study. Atherosclerosis, 1997; 13: 115-125

6) Sawayama Y, Hamada M, Otaguro S, Maeda S, Ohnishi H, Taira Y, Hayashi J: Impact of peripheral arterial disease and acute ischemic stroke. Fukuoka Igaku Zasshi, 2006; 97: 293-301 (in Japanese)

7) Newman AB, Siscovick DS, Manorioet AL: Ankle-arm index as a marker of atherosclerosis in the cardiovascular health study: cardiovascular heart study (CHS), collaborative research group. Circulation, 1993; 88: 837-845

8) Meijer WT, Hoes AW, Rutgers D, Bots ML, Hofman A, Grobbee DE: Peripheral arterial disease in the elderly: The Rotterdam study. Arterioscler Thromb Vasc Biol, 1998; 18: $185-192$

9) Selvin E, Erlinger TP: Prevalence of and risk factors for peripheral arterial disease in the United States: results from the National Health and Nutrition Examination Survey, 1999-2000. Circulation, 2004; 110: 738-743

10) Murabito JM, Evans JC, Nieto K, Larson MG, Levy D, Wilson Peter W.F: Prevalence and clinical correlates of peripheral arterial disease in the Framingham Offspring Study. Am Heart J, 2002; 143: 961-965

11) He Y, Jiang Y, Wang J, Fan L, Li XY, Hu FB: Prevalence of peripheral arterial disease and its association with smoking in a population-based study in Beijing, China. J Vasc Surg, 2006; 44: 333-338

12) Fujiwara $T$, Saitoh $S$, Takagi $S$, Ohnishi H, Ohata J, Takeuchi H, Isobe T, Chiba Y, Katoh N, Akasaka H, Shimamoto K: Prevalence of asymptomatic arteriosclerosis obliterans and its relationship with risk factors in inhabitants of rural communities in Japan: Tanno-Sobetsu study. Atherosclerosis, 2004; 177: 83-88

13) Hozawa A, Ohmori K, Kuriyama S, Shimazu T, Niu kaijun, Watando A, Ebihara S, Matsui T, Ichiki M, Nagato- mi R, Sasaki H, Tsuji I: C-reactive protein and peripheral artery disease among Japanese elderly: the Tsurugaya Project. Hypertens Res, 2004; 27: 955-961

14) Cui R, Kitamura A, Yamagishi $K$, Tanigawa $T$, Imano $H$, Ohira T, Sato S, Shimamoto T, Iso H: Ankle-arm blood pressure index as a correlate of preclinical carotid atherosclerosis in elderly Japanese men. Atherosclerosis, 2006; 184: 420-424

15) McDermott MM, Liu K, Criqui MH, Ruth K, Goff D, Saad MF, Wu C, Homma S, Sharrett R: Ankle-brachial index and subclinical cardiac and carotid disease: the multi-ethnic study of atherosclerosis. Am J Epidemiol, 2005; 162: 33-41

16) Bots ML, De Jong PT, Hofman A, Grobbee DE: Left, right, near or far wall common carotid intima-media thickness measurements: associations with cardiovascular disease and lower extremity arterial atherosclerosis. J Clin Epidemiol, 1997; 50: 801-807

17) Allan PL, Mowbray PI, Lee AJ, Fowkes GR: Relationship between carotid intima-media thickness and symptomatic and asymptomatic peripheral arterial disease. The Edinburgh Artery Study. Stroke, 1997; 28: 348-353

18) Kishihara Y, Furusyo N, Kashiwagi K, Mitsutake A, Kashiwagi Seizaburo, Hayashi Jun: Human T lymphotropic virus type 1 infection influences hepatitis $\mathrm{C}$ virus clearance. J Infect Dis, 2001; 184: 1114-1119

19) Hayashi J, Kishihara Y, Yamaji K, Yoshimura E, Kawakami Y, Akazawa K, Kashiwagi S: Transmission of hepatitis $\mathrm{C}$ virus by health care workers in a rural area of Japan. Am J Gastroenterol, 1995; 90: 794-799

20) Ito J, Dung DT, Vuong MT, Tuyen DG, Vinh LD, Huong NT, Ngoc TB, Ngoc NTB, Hien MT, Hao DD, Oanh LTK, Lieu DT, Fujisawa M, Kawabata M, Shirakawa T: Impact and perspective on chronic kidney disease in an Asian developing country: a large-scale survey in North Vietnam. Nephron Clin Pract, 2008; 109: 25-32

21) Friedewald WT, Levi RI, Fredrickson DJ: Estimation of the concentration of low density lipoprotein cholesterol in plasma without use of the ultracentrifuge. Clin Chem, 1972; 18: 499-452

22) Clairotte C, Retout S, Potier L, Roussel R, Escoubet B: Automated ankle-brachial pressure index measurement by clinical staff for PAD diagnosis in non-diabetic and diabetic patients. Diabetes Care, 2009; 32: 1231-1236

23) Brouwer BG, Visseren FL, Stolk RP, van der Graaf Y: Abdominal fat and risk of coronary heart disease in patients with peripheral arterial disease. Obesity, 2007; 15: 16231630

24) Planas A, Clará A, Pou JM, Vidal-Barraquer F, Gasol A, Moner A, Contreras C, Marrugat J: Relationship of obesity distribution and peripheral arterial occlusive disease in elderly men. Int J Obes Relat Metab Disord, 2001; 25: 1068-1070

25) Nyamdorj R, Qiao Q, Lam TH, Tuomilehto J, Ho SY, Pitkäniemi J, Nakagami T, Mohan V, Janus ED, Ferreira SR: BMI compared with central obesity indicators in relation to diabetes and hypertension in Asians. Obesity, 2008; 16: 1622-1635

26) Arai H, Yamamoto A, Matsuzawa Y, Saito Y, Yamada N, Oikawa S, Mabuchi H, Teramoto T, Sasaki J, Nakaya N, 
Itakura H, Ishikawa Y, Ouchi Y, Horibe H, Shirahashi N, Kita T: Prevalence of metabolic syndrome in the general Japanese population in 2000. J Atheroscler Thromb, 2006; 13: 202-208

27) Lee CG, Carr MC, Murdoch SJ, Mitchell E, Woods NF, Wener MH, Chandler WL, Boyko EJ, Brunzell JD: Adipokines, inflammation, and visceral adiposity across the menopausal transition: a prospective study. J Clin Endocrinol Metab, 2009; 94: 1104-1110

28) Reich LM, Heiss G, Boland LL, Hirsch AT, Wu K, Folsom AR: Ankle-brachial index and hemostatic markers in the Atherosclerosis Risk in Communities (ARIC) study cohort. Vasc Med, 2007; 12: 267-273

29) Han TS, Sattar N, Williams K, Gonzalez-Villalpando C, Lean ME, J Haffner SM: Prospective study of C-reactive protein in relation to the development of diabetes and metabolic syndrome in the Mexico City Diabetes Study. Diabetes Care, 2002; 25: 2016-2021

30) Doi Y, Ninomiya T, Hata J, Yonemoto K, Arima H, Kubo M, Tanizaki Y, Iwase M, Iida M, Kiyohara Y: Proposed criteria for metabolic syndrome in Japanese based on prospective evidence: the Hisayama study. Stroke, 2009; 40: 1187-1194 